Supporting Information

Revealing the Mechanical Bending Mechanisms of Single-Crystalline Rutile $\mathrm{TiO}_{2} \mathrm{Nanowires}^{-}$ Near Room Temperature: Implications for Nanostructured Semiconductors

Qiong Liu, ${ }^{* a}$ Arixin Bo, ${ }^{d}$ Haifei Zhan, ${ }^{a, c}$ Liangzhi Kou, ${ }^{a}$ and Yuantong Gu ${ }^{* a, b}$

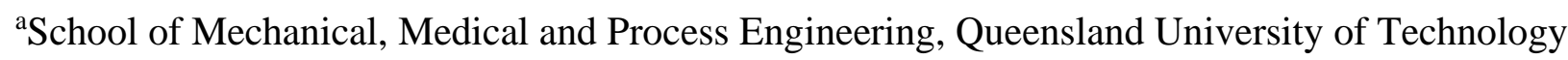
(QUT), Brisbane, Queensland 4001, Australia

${ }^{\mathrm{b}}$ Center for Materials Science, Queensland University of Technology (QUT), Brisbane, Queensland 4001, Australia

${ }^{\mathrm{c}}$ Department of Civil Engineering, Zhejiang University, Hangzhou 310058, China

dINM - Leibniz Institute for New Materials, Saarbrücken 66123, Germany

*Email: yuantong.gu@qut.edu.au; q32.liu@qut.edu.au 


\section{Supplementary Movie}

Movie S1. In situ TEM bending test of a rutile $\mathrm{TiO}_{2}$ nanowire.

\section{Supplementary Figures}
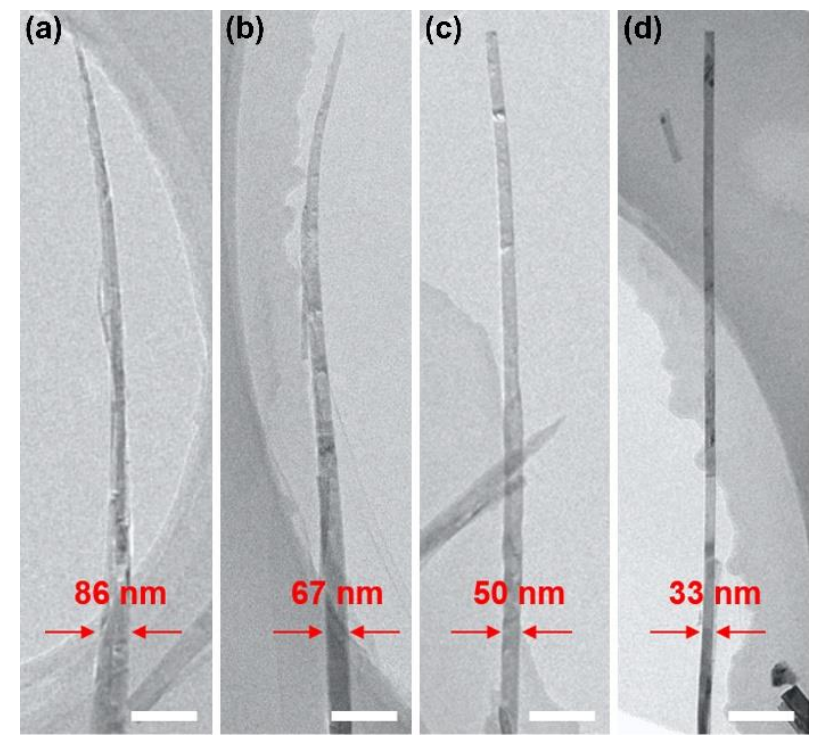

Figure S1. Low-magnification TEM images of the as-prepared rutile $\mathrm{TiO}_{2} \mathrm{NWs}$. (a-d) TEM images of NWs showing roughly the same length from the top. The thickness of the NWs at the same location from the top decrease gradually from Panels a-d. Thick NWs (diameters: $>40 \mathrm{~nm}$ ) show tapered morphologies (Panels a-c); The thin NW (diameter: $\sim 33 \mathrm{~nm}$ ) has an even diameter (Panel d). Scale bar: 200nm. 


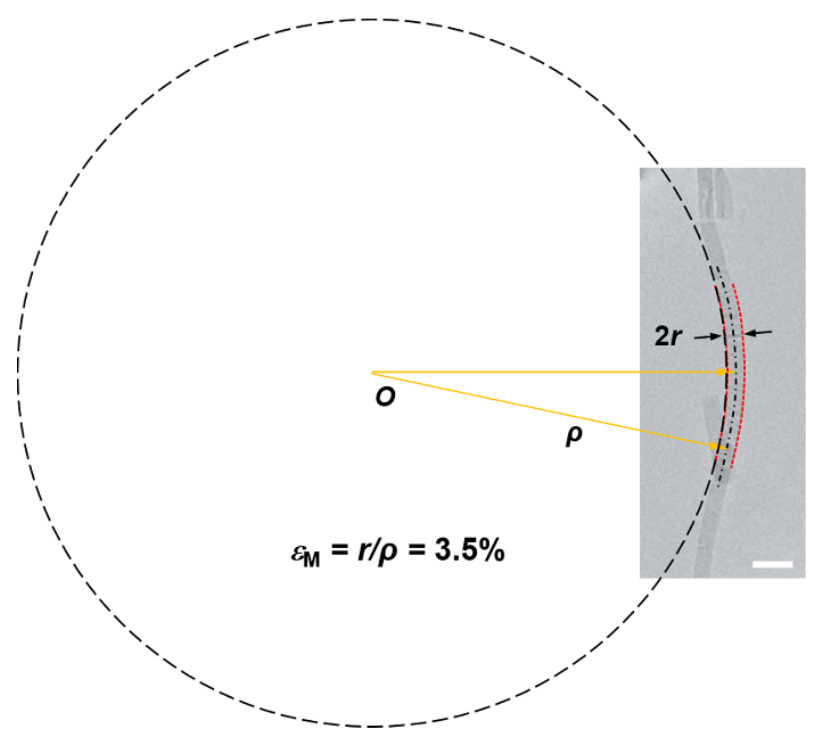

Figure S2. Schematic of calculating the maximum bending strain in the most strained segment of the NW. A circle is drawn to trace the middle line along the most strained segment of the NW, whose radius can be considered as the radius $(\rho)$ of the local bending curvature. After measuring the radius $(r)$ of the NW and $\rho$, the bending strain of this segment can be calculated according to the equation, $\varepsilon_{\mathrm{M}}=r / \rho$. Scale bar: $20 \mathrm{~nm}$. 


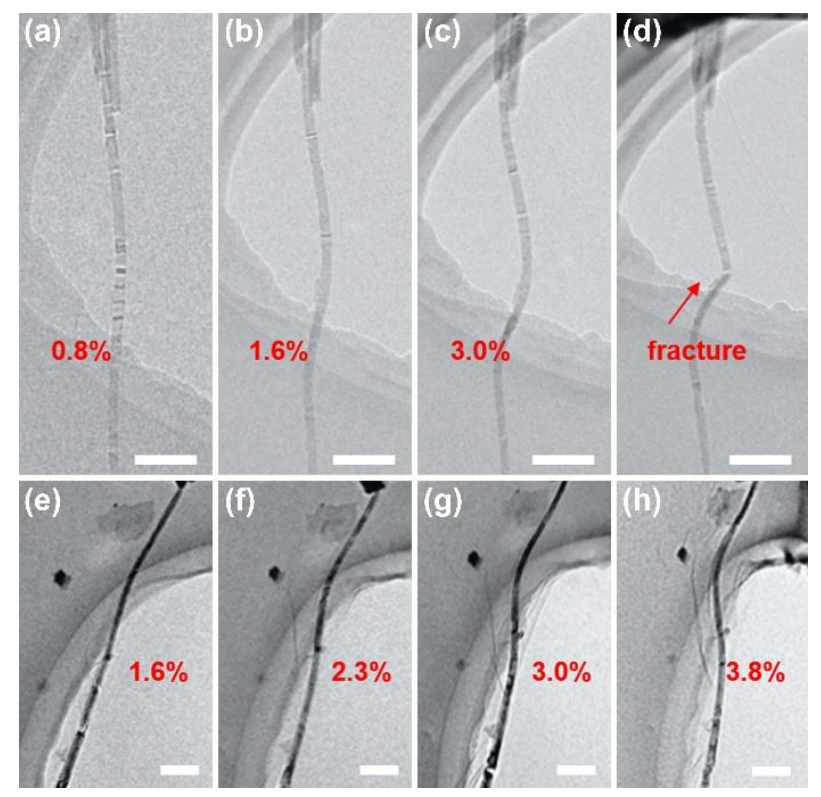

Figure S3. In situ bending tests of two individual rutile $\mathrm{TiO}_{2} \mathrm{NWs}$. (a-d) TEM images showing that the NW with a diameter of $\sim 29 \mathrm{~nm}$ can withstand a maximum bending strain of $\sim 3.0 \%$. (e-h) TEM images showing that the NW with a diameter of $\sim 34 \mathrm{~nm}$ can withstand a bending strain of $3.8 \%$. 


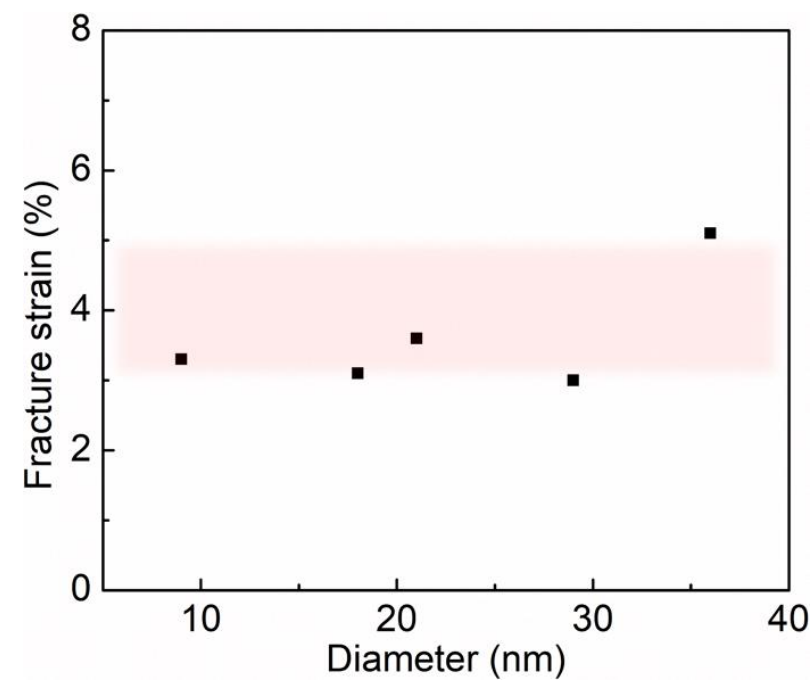

Figure S4. Summary of the fracture strains of the tested thin rutile $\mathrm{TiO}_{2} \mathrm{NWs}$ with variant diameters.

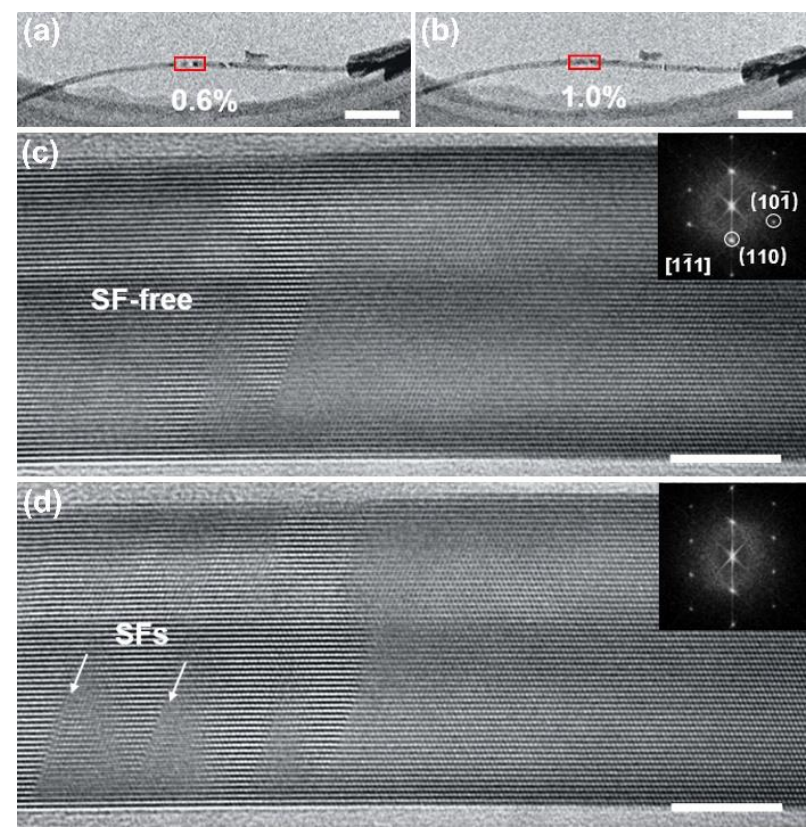

Figure S5. In situ bending tests of a rutile $\mathrm{TiO}_{2} \mathrm{NW}$ with a diameter of $\sim 27 \mathrm{~nm}$. (a,b) Lowmagnification TEM images of the NW with bending strains of $0.6 \%$ and $1.0 \%$, respectively. (c,d) HRTEM images of the same framed area in Panels a and $b$, showing a SF-free structure and two SFs, respectively. Scale bar: $200 \mathrm{~nm}$ in Panels a and b; $10 \mathrm{~nm}$ in Panels $\mathrm{c}$ and $\mathrm{d}$. 